\title{
The Design and Implementation on Distributed System
}

\author{
Xin Sui ${ }^{1,2}$, Zetian Zhang ${ }^{1}$, Yan Zeng ${ }^{1}$, Dan $\mathrm{Liu}^{1}, \mathrm{Li} \mathrm{Li}^{1, *}$, Liu Liu ${ }^{3}$ \\ ${ }^{1}$ Changchun University of Science and Technology, 130022, China. \\ ${ }^{2}$ Jilin Provincial Institute of Education, 130022, China \\ ${ }^{3}$ Baicheng Ordnance Test Center, 130022, China \\ *Corresponding Author: Li Li, email: ll@cust.edu.cn, tel: 85582352
}

Keywords: Distributed system, LSM merging tree, Election algorithm, roadblock detection, Arduino

\begin{abstract}
The paper achieves the traveling balance control in exploratory behavior by fuzzy logic method, uses the least squares fitting to realize the corner detection and realizes the roadblock detection by designing the Barrier Predictive Function Model. The paper designs a distributed system based on block storage and electoral computing model which provides high reliability storage and computing services. This paper proposes the BUS communication model, achieves the storage and computing node's selection by LSM merging tree and Bully algorithm. The real-time ultrasonic roadblock detection information data obtained by Arduino detector is analyzed based on the distributed system and finally, the detection of environmental map is realized.
\end{abstract}

\section{INTRODUCTION}

Map detection is a prerequisite for complex tasks such as gathering, path planning, risk detection and etc. An effective exploratory strategy can coordinate detectors to complete cognitive and perceptual processes for unknown environments more quickly and efficiently [1]. The construction of the environmental map is a very influential research direction. The operating space is block in many cases, the detector firstly needs to detect its surroundings and build an environment map. Finally, the follow-up research can be token with the help of the map information [2]. The research on distributed system is an important content of today's academic circles. The idea of normalizing the hardware resources through software enriches its theory increasingly. Based on the actual needs of the research subject, the paper explores a practical solution to map detection methods on distributed system which can improve the reliability and durability of available resources.

\subsection{Detection algorithm design}

The researchers achieve the triangular formula modeling by placing ultrasonic modules on left, right and front sides of the detector and installing a rotating speed steering gear on the front side and uses fuzzy logic for deviation detection. Detector chooses the minimum distance between two sides of the wall as the reference direction, the front ultrasonic module will deflect 45 degrees to the reference direction. In the ideal state, the model is isosceles right triangle as shown in Figure 1.

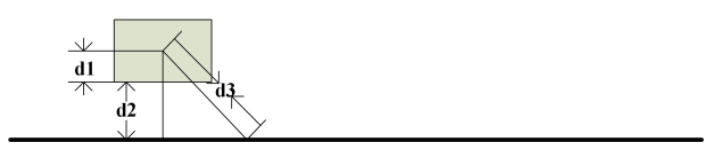

Figure1: Ideal route model
The sum of the width of the detector itself $d_{1}$ and the distance measured by ultrasonic wave $d_{2}$ is $\sqrt{2}$ times as large as $d_{3}$. There is a certain measurement error in the actual scene. When there exists the moving track deviation, it is necessary to take $d_{1}, d_{2}$ and $d_{3}$ values into the fuzzy logic system to achieve track correction. Figure 2 is a detector state that deviates from the orbit.

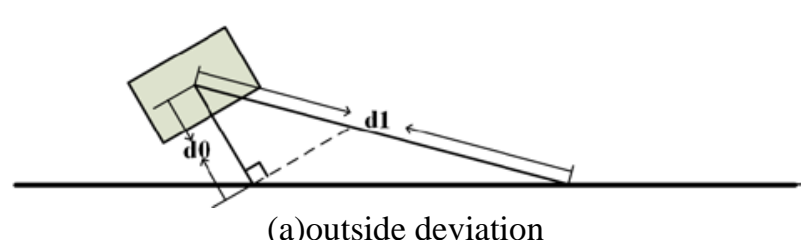

(a)outside deviation

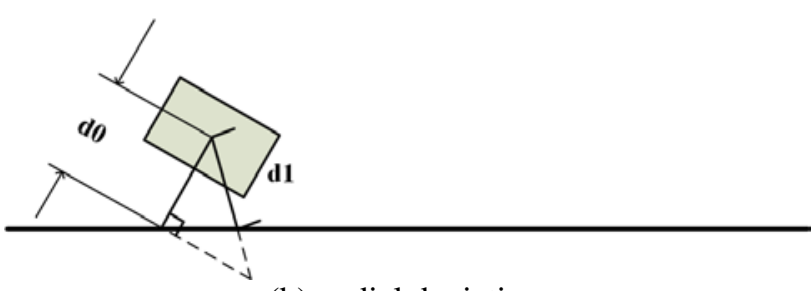

(b)medial deviation

Figure 2: The deviation state of detecting orbit

In the case of outside deviation, the measured distance $\mathrm{d}_{0}$ multiples of $\sqrt{2}$ is far less than the measured distance $\mathrm{d}_{1}$. In this paper, the relation between the distance measured and the ideal distance is used as the input parameter of the fuzzy logic system, the outside deviation formula is shown as follows:

$$
\text { input }=\frac{d_{0} * \sqrt{2}}{d_{1}} * 100 \%
$$

In the case of innerdeviation, the measured distance 
$d_{1}$ is less than the measured distance $d_{0} * \sqrt{2}$. The paper uses the distance percentage as the input parameter of fuzzy logic system either. The deviation formula is shown as follows:

$$
\text { input }=\frac{d_{1}}{d_{0} * \sqrt{2}} * 100 \%
$$

The membership function of the fuzzy logic is shown in Figure 3. The dotted line represents the percentage of inner deviationcoefficients, the solid line represents the percentage of outside deviationcoefficients. The current source outputs 1 represents the detectormotor current is applied to the reverse direction, the current source 0 represents an electric is current applied to the detecting reference direction motor.

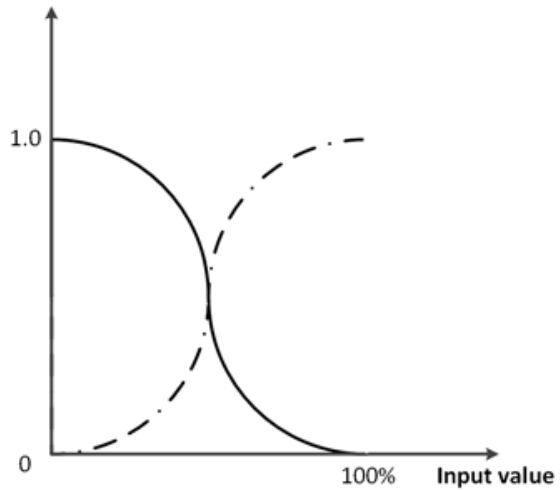

Figure 3: Membership function of balance travel

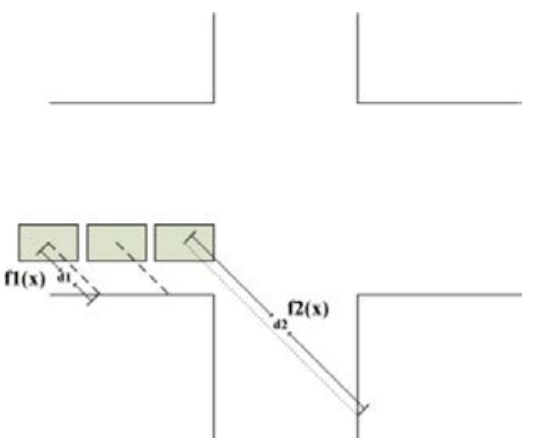

Figure 4: The schematicof curve turningalgorithm

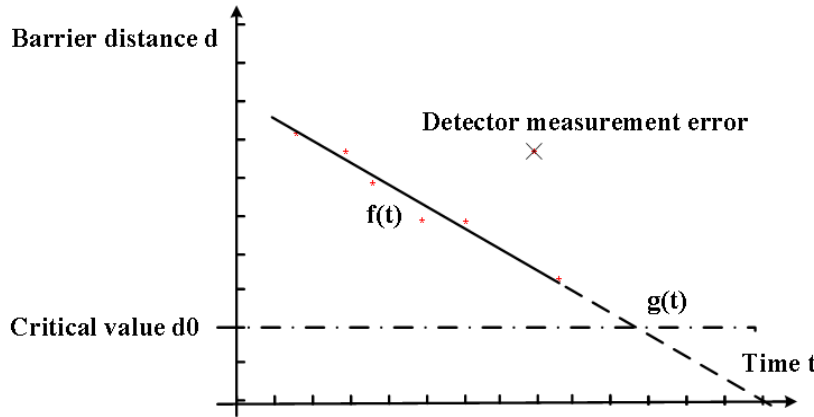

(a)Prediction model of roadblock
The essence of curve turning algorithm is the problem of path identification at crossroads (Or a fork). In this paper, the curve recognition is realized by comparing two gradient functions with the least square fitting. Take the crossroads as an example, the gradient change of the front detector's ultrasonic information function means that the traffic curve appears at present. The road map and the gradient function are shown in figure 4:

Based on the principle, the proposed formula is:

$$
\left\{\begin{array}{l}
f_{1}(x)=b_{0} \\
f_{2}(x)=-\sqrt{2} x+b_{1}
\end{array}\right.
$$

The measured function $\mathrm{f}_{1}(\mathrm{x})$ increases when the fork appeared in the path of the detector A. Under unknown conditions, theincrease may be caused by a measurement error of the chip or a road wobble difference. Therefore, the difference in the $K$ value between the primary function $\mathrm{f}_{2}(\mathrm{x})$ and the $\mathrm{f}_{1}(\mathrm{x})$ should be checked by obtaining multiple raised measurements. In this paper, the $b_{1}$ constant interpolation is set to greater than or equal to 2 times $b_{0}$. It's reason is considering the temporary space in which the detector will occupy a certain area during the turning job.

In this paper, the ultrasonic ranging method is used as the main method to obtain the surrounding road condition and the detector may causesome measurement errors in the rough road condition. Therefore, the data fitting method is used to merge the information in a certain range and finally the prediction function is generated. The function model is shown in figure 5 (a), $\mathrm{f}$ $(\mathrm{t})$ is a fitting function of obstacle detection information, in which the measured values outside the set will become the measurement error points and then can be excluded. The $g(t)$ is a prediction function generated by the time advance. When the detector reaches the critical value in next second, it will take the obstacle avoidance strategy. In this paper, the obstacle avoidance strategy is by selecting the information on both sides of the barrier and the maximum value is the turning direction. The strategy is illustrated in Figure 5 (b)

Figure 5: Ultrasonic road block detection

\subsection{Bus distributed shared memory}

Inspired by the development of multi-processor structures, the distributed shared memory (DSM) can be concluded in three ways, which are chip memory, based on bus and ring [3]. In the scene of asynchronous transmission pattern (ATM) based on chip DSM exists a large number of processing units not only its construct is extremely complex but also it'sexpensive andimpractical. The DSM based on bus can realize storage allocation and control access by bus arbitration mechanism. The DSM based on ring can divide independent address space into 
dedicated and shared area, where the shared area is synchronized to all machines. The general structure of DSM based on bus is shown in Figure 6. The shared memory represents logical view of user's perspective; the local memory is a physical view. The shared units represent the unit size, and it can be moved among different local memories.

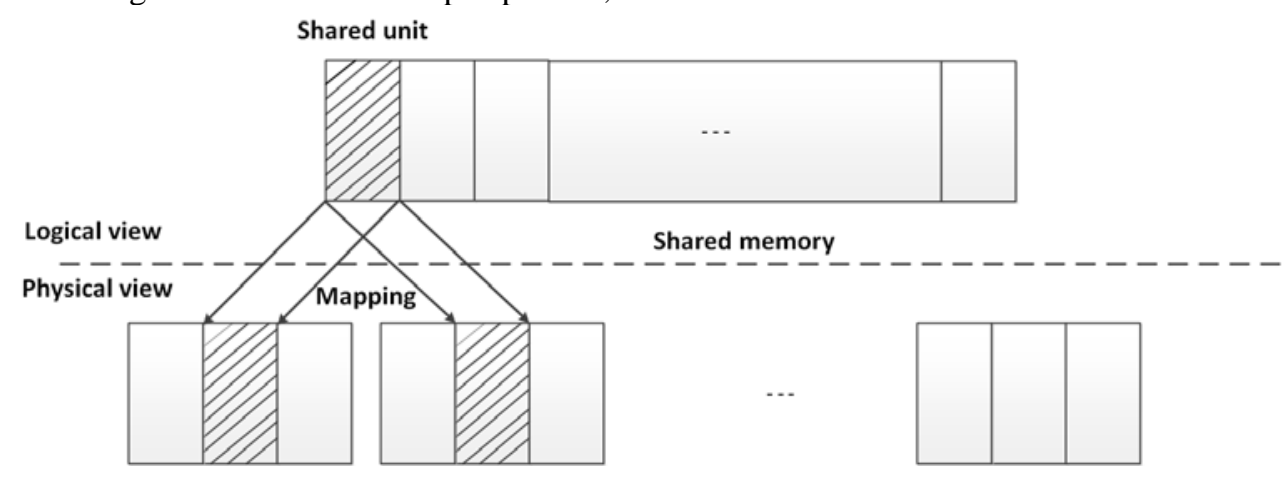

Figure 6: Distributed shared memories

\subsection{Analysis of storage node based on LSM}

Two problems of distributed system storage are:storage-related algorithms and data position access. It's essential to provide good storage design and efficient storage spacefacing a set of frequent writing and query data at any time. Patrick O'Neil1 proposed the structure of the log structure merge tree (LSM tree) in his pager, it cascaded memory and disk components in an efficient way with the merge sort method [4]. The tree set is $\left\{\mathrm{C}_{0}, \mathrm{C}_{1}, \ldots, \mathrm{C}_{\mathrm{k}}\right\}$, where $\mathrm{C}_{0}$ stores in memory buffer while $\mathrm{C}_{\mathrm{i}(\mathrm{i} \neq 0)}$ store in disk array. Though the tree $\mathrm{C}_{1}$ resides in disk, the page node which cited frequently still reside in memory buffer area. The content is inserted into $\mathrm{C}_{0}$ firstly and then is moved to $\mathrm{C}_{1}$. Any index entry is hit to accessin $\mathrm{C}_{0}$ and then within $\mathrm{C}_{1}$. Comparing with diskinjection, the cost of inserting content into memory-resident tree $\mathrm{C}_{0}$ is extremely low. However, memory costs and capacity limits the structuresize. When the tree $C_{0}$ is close to the maximum allocation threshold, it triggers the scrolling merge event to remove the lowhit rate element from the $\mathrm{C}_{0}$ tree by hot and cold zone method to merge them into the $\mathrm{C}_{1}$ tree. Similarly, the structure of LSM tree allows defining multiple components $\mathrm{C}_{0}, \mathrm{C}_{1}, \mathrm{C}_{2} \ldots \mathrm{C}_{\mathrm{K}-1}, \mathrm{C}_{\mathrm{k}}$, where $\mathrm{C}_{0}$ represents memory resident, others components are disk residents. Each smaller component $\mathrm{C}_{\mathrm{i}-1}$ will be distributed equally to the high component $C_{i}, C_{i}+1 \ldots C_{k}$ through the asynchronous scrolling merge method when it is close to the maximum allocation threshold.

\subsection{Analysis of computing node based on Bully}

Based on taking the system development model and data migration into consideration, the paper realizes election of computing service by Bully algorithm. This algorithm means that it will initiate the election when a process finds the coordinator no longer response to the request [5]. The election procedure is shown as following:

s1. The process $\mathrm{P}$ sends election information to all processes whose numbers are larger than it.

s2. If no one responds, $\mathrm{P}$ will win as a coordinator.

s3. If the number process larger than $\mathrm{P}$ responses, the responder takes over, work $\mathrm{P}$ is finished.

In this paper, the algorithm is applied in distributed systems to provide data computing services to detectors.
After the ultrasonic data set is sent to the system and stored in the DSM, the system will elect the calculation node according to the Bully algorithm, the computational resource node measures formula is shown in formula 4.

$$
\text { measure }=\frac{n_{-} \text {memory }}{c_{-} \text {memory }} * \frac{n_{-} P E}{c_{-} P E} * 100 \%
$$

The n_memory is the RAM of node to be selected, c _memory is the total RAM of cluster. n_PE is the number of to be selected node's processing unit core, and $\mathrm{C} \_\mathrm{PE}$ is the number of the total processing unit core.

\section{SYSTEM DESIGN AND IMPLEMENTATION}

\subsection{Distributed system design}

The distributed system is divided into two modules, DSM and election computing, the researchers useKVM virtualization technology to structure multiple virtual servers and build a virtual network based on the physical server set.The paper proposes a BUS communication model for global information synchronization based on the traditional distributed data transmission model (PUBSUB/SURVEY/REQREP/PAIR).The BUS model stores the distributed data for the entire topology network.Based on the idea of transfer and replication in distributed systems, we consider that one node failure does not cause the DDoS.The solution is to distribute the data in multiple nodes by performing the data multilayer backup. In the SURVEY model, the same information block is sent to the relevant nodeindividually. Its data access patternis sending the inquiry information to the relevant node by the central control node and obtains the data on nearest route node and feeds back to the visitor.

In this paper, we realize the merging and storage of the data node storage and the election of computing nodes through the researchon LSM merging tree and Bully algorithm. The computing node accesses the detector datasets through DSM and separately sends the results to the detector and log storage node which plots the result. The network access forwarding method is used to achieve communication between the detector and the systemwhich is shown in Figure 7 (a). The hierarchical 
design of the distributed system is shown in Figure 7(b). The researchers implement the fuzzy rules and the least squares coding on the basis of the computing services

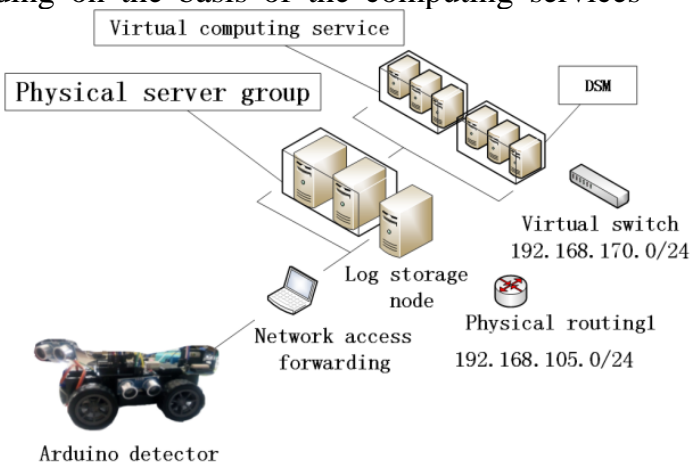

(a) Distributed System PhysicalArchitecture and application interface provided by the system. The detector utilizes both to achieve deviationdetection and curve recognition.

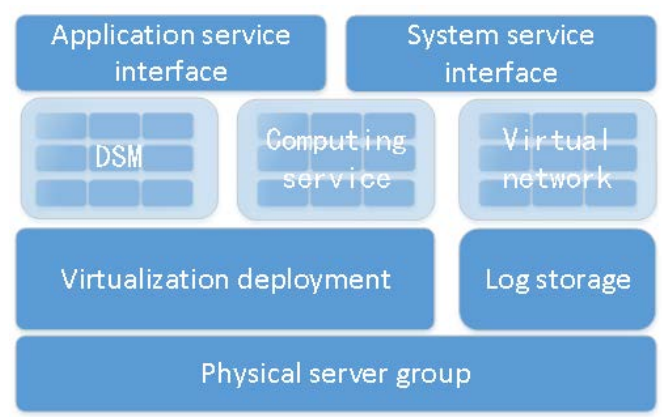

(b) Distributed System Logical Architecture

Figure 7: Distributed system architecture

\subsection{Detector circuit design}

In the experiment section, the detector is designed as follows: Using L298N DC to achieve back drive motor propulsion and front motor control steering. Using HC-SR04 ultrasonic module to realize physical information ranging and using the GPRS chip to submit the distance information to the distributed system. The system calculates the distance information and the result message is sentback to the Arduino chip. Finally, the Arduino chip realizes the control of the motor indirectly through the input of the PWM signal to the L298N. The detector circuit design and physical display is shown in Figure 8. The detector and the distributed system communicate by TCP mode. The Arduino chip analyzes the instructions in the received message and passesthe command to the settled pin in digital signal mode.

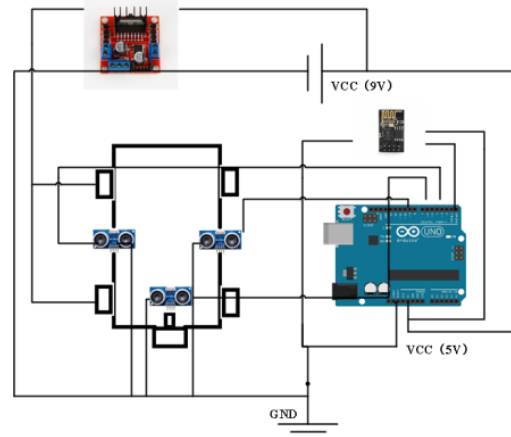

(a) $\overline{\text { Circuit design }}$

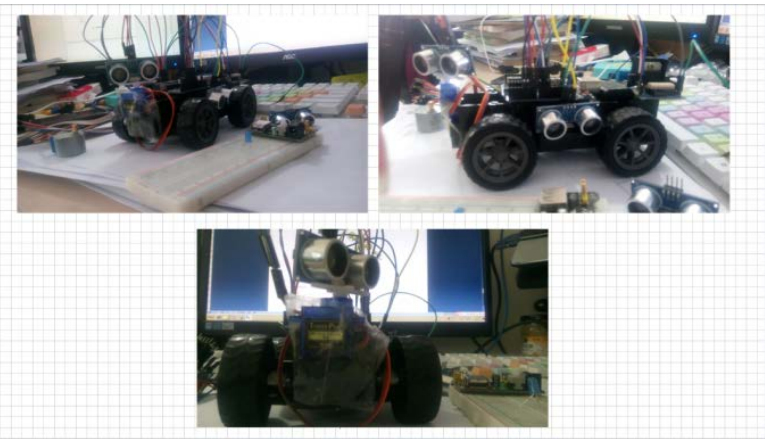

(b) Detector physical diagram

Figure 8: Schematic of detector

\section{EXPERIMENTAL RESULTS AND ANALYSIS}

\subsection{Detector data acquisition}

The experiment data are derived from the experimental site acquisition. In this experiment, the detector collects the ultrasonic distance information and the angle information of the steering gear and the lateral ultrasonic distance information. The data of the balancing route and the detour route datain the actual testfield are shown in Table 1.

Table 1: data of detector path

(a)Balanced route data

\begin{tabular}{|c|r|r|c|}
\hline $\begin{array}{l}\text { Front } \\
\text { distance }\end{array}$ & \multicolumn{1}{|l|}{$\begin{array}{l}\text { Left } \\
\text { distance }\end{array}$} & $\begin{array}{l}\text { Right } \\
\text { distance }\end{array}$ & $\begin{array}{l}\text { Steering } \\
\text { angle }\end{array}$ \\
\hline 41.41 & 179.13 & 30.33 & $45^{\circ}$ \\
\hline 42.03 & 179.04 & 29.97 & $45^{\circ}$ \\
\hline 43.13 & 179.29 & 30.15 & $45^{\circ}$ \\
\hline 42.25 & 180.04 & 30.94 & $45^{\circ}$ \\
\hline 42.82 & 180.53 & 29.23 & $45^{\circ}$ \\
\hline 42.43 & 178.15 & 30.51 & $45^{\circ}$ \\
\hline 55.74 & 201.01 & 32.71 & $45^{\circ}$ \\
\hline 55.66 & 200.94 & 33.01 & $45^{\circ}$ \\
\hline 55.69 & 201.25 & 33.80 & $45^{\circ}$ \\
\hline 55.34 & 199.11 & 31.74 & $45^{\circ}$ \\
\hline 54.57 & 199.22 & 33.87 & $45^{\circ}$ \\
\hline 55.30 & 200.94 & 31.51 & $45^{\circ}$ \\
\hline 42.88 & 180.67 & 30.50 & $45^{\circ}$ \\
\hline
\end{tabular}

(b)Curve routing data

\begin{tabular}{|c|c|c|c|}
\hline $\begin{array}{l}\text { Front } \\
\text { distance }\end{array}$ & $\begin{array}{l}\text { Left } \\
\text { distance }\end{array}$ & $\begin{array}{l}\text { Right } \\
\text { distance }\end{array}$ & $\begin{array}{l}\text { Steering } \\
\text { angle }\end{array}$ \\
\hline 42.16 & 178.13 & 31.01 & $45^{\circ}$ \\
\hline 42.25 & 179.04 & 30.96 & $45^{\circ}$ \\
\hline 341.16 & 179.51 & 30.94 & $45^{\circ}$ \\
\hline 398.31 & 178.48 & 31.31 & $45^{\circ}$ \\
\hline 395.17 & 179.94 & 30.50 & $45^{\circ}$ \\
\hline- & - & - & - \\
\hline- & - & - & - \\
\hline- & - & - & - \\
\hline 253.9 & 30.96 & 180.22 & $45^{\circ}$ \\
\hline- & 30.96 & 180.22 & $20^{\circ}$ \\
\hline- & 30.96 & 180.22 & $-10^{\circ}$ \\
\hline 43.18 & 30.87 & 178.23 & $-45^{\circ}$ \\
\hline 43.96 & 31.03 & 177.12 & $-45^{\circ}$ \\
\hline
\end{tabular}




\section{\begin{tabular}{|l|l|l|l|}
\hline 42.05 & 179.21 & 29.83 & $45^{\circ}$ \\
\hline 41.90 & 180.87 & 29.31 & $45^{\circ}$ \\
\hline
\end{tabular}}

\subsection{Communication data within a distributed system}

After the central server group receives the data collected by the detector, it is packaged in JSON format

\begin{tabular}{|l|l|l|l|}
\hline 44.06 & 31.29 & 177.16 & $-45^{\circ}$ \\
\hline 42.48 & 30.30 & 178.51 & $-45^{\circ}$ \\
\hline
\end{tabular}

and then distributed by SURVEY/BUS mode. The distributed system internal communication is JSON format either. The data is shown in Figure 9.
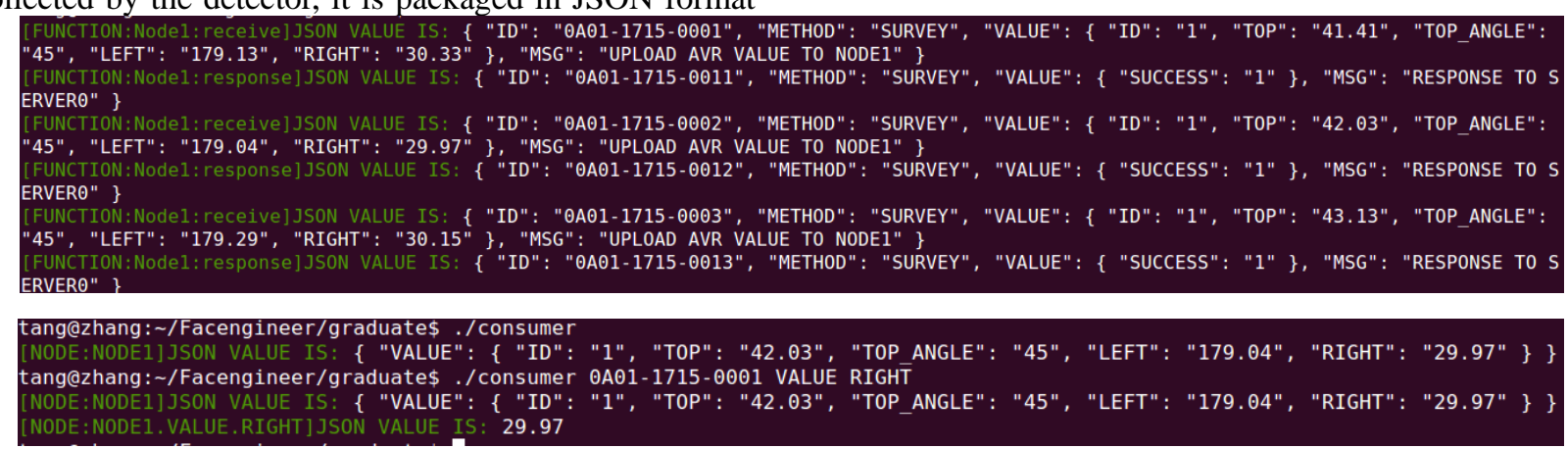

Figure 9: MemoryNode data

\subsection{Result analysis}

As the research method used in this experiment, the researchers compare the probe results with the experimental site and finally the experimental results are verified. The drawing process parses the ID file of the corresponding detector and finally manages to hit the canvas. At the same time, the purpose of azimuth controlling is achieved by introducing compass model to drawing system. The balancing travel strategy is illustrated in Figure 10 (a), The curve detour strategy is illustrated in Figure 10 (b), The experimental results are shown in Figure 10 (c).

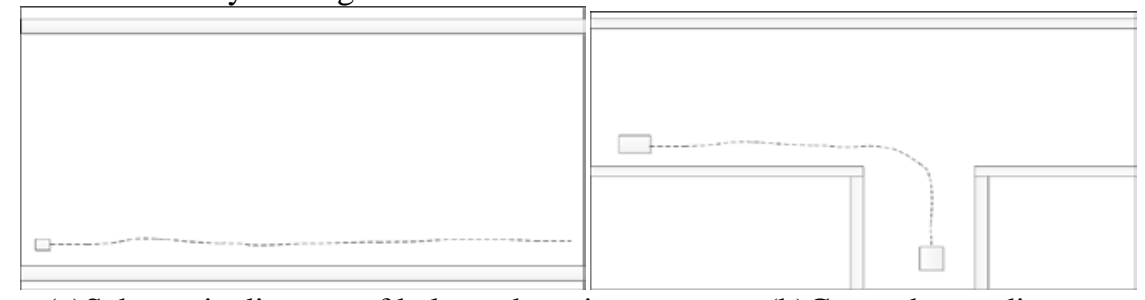

(a)Schematic diagram of balanced motion

(b)Curve detour diagram
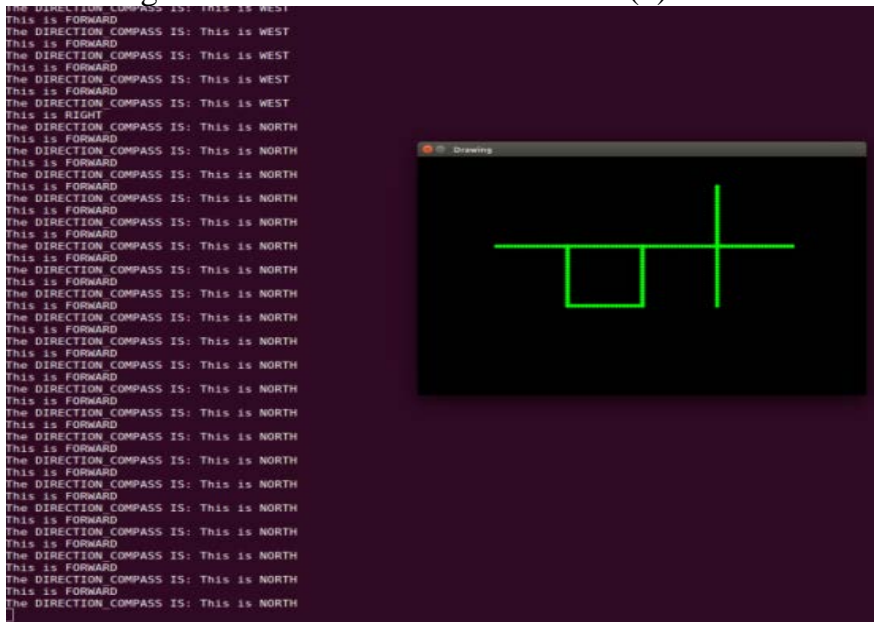

(c)Experimental result chart

Figure 10: Experimental drawing results

\section{CONCLUSIONS}

In this paper, Arduino map detectors are used on the basis of distributed system. Its purpose is to solve the service crashes during traditional remote control process by usingdistributed system which provides high concurrency and resource reliability service. We propose the BUS communication model to expand traditional PUBSUB/SURVEY communication model. The paper researches and implements the detection to simple map construction environment and provides theoretical reserves for future complex environmental analysis.

\section{ACKNOWLEDGMENTS}

This work was supported in part by the science and technology project of "13th Five-Year" planning of the Education Department of Jilin Province (JJKH20170631KJ); the key project of "13th Five-Year" planning of the Education Science of Jilin Province 
(ZD16024); the Key Science and Technology Project of Jilin Province (20160204019GX).

\section{REFERENCES}

[1] Wang H.T., 2014. Multi-robot Exploration and Mapping in Unknown Environment. ZhengZhou university.

[2] Yan Y., 2010. Distributed map integration ofr muti-robot exploration. ShangHai JiaoTong university.

[3] Tanenbaum, A. S., \& Steen, M. V., 2002. Distributed Systems: Principles and Paradigms. Tsinghua University Press. [4] O’Neil, P., Cheng, E., Gawlick, D., \& O’Neil, E., 1996. The log-structured merge-tree (LSM-tree). Acta Informatica, 33(4): 351-385.

[5] Garcia-Molina, 1982. Elections in a Distributed Computing System. IEEE Transactions on Computers, C-31(1): 48-59. 